\title{
LUT
}

Lappeenranta

University of Technology

\section{Automated Segmentation of Nanoparticles in BF TEM Images by U-Net Binarization and Branch and Bound}

Zafari Sahar, Eerola Tuomas, Ferreira Paulo, Kälviäinen Heikki, Bovik Alan

This is a Final draft version of a publication

published by Springer, Cham

in Computer Analysis of Images and Patterns. CAIP 2019.

DOI: $10.1007 / 978-3-030-29888-3 \_10$

Copyright of the original publication: (C) 2019 Springer Nature Switzerland AG.

Please cite the publication as follows:

Zafari, S., Eerola, T., Ferreira, P., Kälviäinen, H., Bovik, A., Automated Segmentation of Nanoparticles in BF TEM Images by U-Net Binarization and Branch and Bound, Computer Analysis of Images and Patterns, Springer Lecture Notes in Computer Science, LNCS Vol. 11678, pp. 113-125, 2019, Proceedings of the 18th International Conference on Computer Analvsis of Imaaes and Patterns (CAIP 2019). Salerno. Italv. 2019. DOI: https://doi. 


\title{
Automated Segmentation of Nanoparticles in BF TEM Images by U-Net Binarization and Branch and Bound
}

\author{
Sahar Zafari ${ }^{1}$, Tuomas Eerola ${ }^{1}$, Paulo Ferreira ${ }^{3,4,5}$, Heikki Kälviäinen ${ }^{1}$, and Alan \\ Bovik $^{2}$ \\ 1 Computer Vision and Pattern Recognition Laboratory (CVPR), Department of Computational \\ and Process Engineering, School of Engineering Science, Lappeenranta-Lahti University of \\ Technology LUT, Finland \\ ${ }^{2}$ Laboratory for Image and Video Engineering (LIVE), Department of Electrical and Computer \\ Engineering, The University of Texas at Austin, Austin, Texas 78712, USA \\ 3 Materials Science and Engineering Program, The University of Texas at Austin, Austin, Texas \\ 78712, USA \\ 4 INL-International Iberian Nanotechnology Laboratory, Av. Mestre José Veiga s/n, 4715-330 \\ Braga, Portugal \\ 5 Mechanical Engineering Department and IDMEC, Instituto Superior Técnico, University of \\ Lisbon, Av. Rovisco Pais, 1049-001 Lisboa, Portugal
}

\begin{abstract}
Transmission electron microscopy (TEM) provides information about Inorganic nanoparticles that no other method is able to deliver. Yet, a major task when studying Inorganic nanoparticles using TEM is the automated analysis of the images, i.e. segmentation of individual nanoparticles. The current state-ofthe-art methods generally rely on binarization routines that require parameterization, and on methods to segment the overlapping nanoparticles (NPs) using highly idealized nanoparticle shape models. It is unclear, however, that there is any way to determine the best set of parameters providing an optimal segmentation, given the great diversity of NPs characteristics, such as shape and size, that may be encountered. Towards remedying these barriers, this paper introduces a method for segmentation of NPs in Bright Field (BF) TEM images. The proposed method involves three main steps: binarization, contour evidence extraction, and contour estimation. For the binarization, a model based on the U-Net architecture is trained to convert an input image into its binarized version. The contour evidence extraction starts by recovering contour segments from a binarized image using concave contour points detection. The contour segments which belong to the same nanoparticle are grouped in the segment grouping step. The grouping is formulated as a combinatorial optimization problem and solved using the well-known branch and bound algorithm. Finally, the full contours of the NPs are estimated by an ellipse. The experiments on a real-world dataset consisting of 150 BF TEM images containing approximately 2,700 NPs show that the proposed method outperforms five current state-of-art approaches in the overlapping NPs segmentation.
\end{abstract}

Keywords: segmentation, image processing, concave points, branch and bound, overlapping, nanoparticles, TEM images. 


\section{Introduction}

Inorganic nanoparticles (NPs) exhibit numerous properties that make them of interest to the research and industrial community [9:10411]. Numerous techniques for characterizing Inorganic NPs exist, and Transmission electron microscopy (TEM) represents one of the most appealing options because it can provide information about a broad range of their features, such as size, shape, texture, crystal structure, and composition. No other technique can provide as much information as TEM, primarily due to ability of the modern instruments to achieve both high contrast, high resolution, and diffraction composition [16]. However, studies of Inorganic NPs using TEM have suffered from poor statistical quality due to the difficulty of their segmentation in the image processing step. Although, the recent studies have made significant progress towards remedying the problem of inaccurate segmentation and the resultant poor statistical information [624|20], segmenting Inorganic NPs in TEM micrographs still rely on either global thresholding or local thresholding [5|2]. Global thresholding [137] has performed successfully in the past in some limited cases where the NPs are clearly distinguishable form background [20]. However, nonuniform backgrounds generally prevent this binarization technique from succeeding. The end result for global thresholding is numerous false negatives and some false positives resulting from a poor binarization. Local thresholding [12|18|1] supposedly has led to improved segmentation results in the presence of nonuniform backgrounds. However, this binarization technique also reproducibly fails to distinguish between the background and the NPs. A recent study [6] has proposed the use of variance hybridized mean local thresholding as a binarization routine, and this method shows promising results. When compared to the baseline of global thresholding, this novel binarization routine effectively eliminates false negatives while greatly reducing false positives, usually providing excellent segmentation. However, variance hybridized mean local thresholding contains a weakness in that it requires an optimized set of parameters to achieve a good binarization. Thus, one must specify values these parameters for each image in dataset in order to use the approach.

In this study, we advance the state-of-the-art by utilizing a binarization technique that does not require parameterization, as well as providing a superior technique for the segmentation of individual NPs from regions of nanoparticle overlap. We propose to use a binarization method based on the convolutional neural networks. The binarization is formulated as a semantic segmentation task in which each pixel in the image is assigned to an object class, the foreground and the background, and is implemented based on the well-known U-Net architecture [17]. We integrate the U-Net based binarization to our segmentation framework for overlapping NPs [24 25] that utilize concave points in the contours of nanoparticle bundles. We demonstrate that the U-Net based binarization produces accurate separation of NPs and background without causing fluctuations to the nanoparticle contours. This makes it possible to detect the concave points robustly and further allows accurate segmentation of individual NPs. We show that the proposed method outperforms five existing methods [6|20|22 26 14] with higher NP detection rate and segmentation accuracy. 


\section{Proposed Method}

The proposed method is summarized in Fig. 1. Given an input grayscale image, the segmentation follows three sequential stages: binarization, contour evidence extraction, and contour estimation. First, the binarization of the image is performed using the well-known encoder-decoder deep framework called U-Net [17]. Second, the contour evidence extraction aims to find and inference the visible contour of each nanoparticle. This involves two separate tasks: contour segmentation and segment grouping. In the contour segmentation the contour segments are recovered from the binarized image using concave point detection and segment grouping, whereby contour segments belonging to the same nanoparticle are grouped by utilizing the branch and bound algorithm [24]. Finally, the contour evidences are utilized by ellipse fitting to estimate the full contours of the NPs.

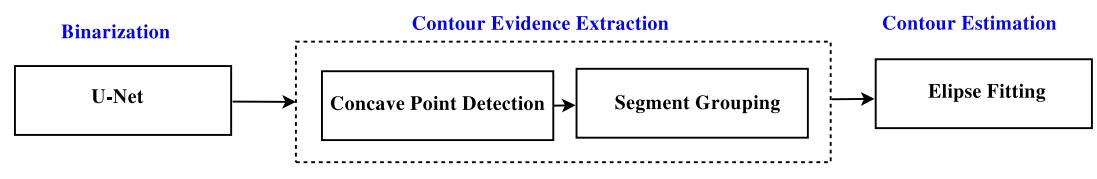

Fig. 1: Proposed segmentation method.

\subsection{Binarization}

The proposed method is organized in a sequential manner where the performance of each module directly impacts the performance of the next step. Binarization is a key step in computation flow that can directly affects the performance of contour evidence extraction. Due to uneven illumination, contrast variation, non-uniform background, and the likely presence of noise in NP images, the binarization is a challenging task. To this end, the binarization routine is formulated as a pixel classification problem that is performed by a convolutional encoding-decoding framework [21]. In particular, it is implemented by the U-Net architecture proposed by Ronneberger et al. [17]. The U-Net contains several layers of convolutional encoders and decoders, followed by the final pixelwise classification layer. Each encoder layer is composed of duplicated $3 \times 3$ convolution operations followed by a rectified linear unit (ReLU). Following that, the encoder layers downsample the feature maps using a $2 \times 2$ max pooling operation with stride 2 . To avoid spatial information lost during downsampling, the encoder feature maps are up-sampled and summed to the corresponding decoder feature maps and passed to the next layer after rectification in the decoder layers. The final layer is $1 \mathrm{x} 1$ convolution to map each feature vector to the desired classes. To classify each pixel and to ensure that all predicted pixels are in the range $[0,1]$ the sigmoid activation function is applied at the output layer.

The loss function for training the network was defined based on the Dice coefficient [27]. Given the prediction $O_{p}$ and the ground truth $O_{g}$ the dice coefficient (DSC) 
measures the similarity as follows:

$$
D S C=\frac{2\left|O_{p} \cap O g\right|}{\left|O_{p}\right|+|O g|} .
$$

The higher the DSC value, the greater the similarity. Since the training aims to minimize the loss function we instead used the negative dice coefficient (-DSC).

\subsection{Concave Point Detection}

Concave point detection has a pivotal role in contour segmentation and provides an important cue for further object segmentation (contour estimation). The main goal of the concave point detection is to find concave locations on the object boundaries and to utilize them to segment the contours of overlapping objects in such a way that each contour segment contains edge points from one object only [23].

Polygonal approximation is a well-known method to represent the objects contours by a sequence of dominant points. It can be used to reduce complexity, to smooth object contours, and to avoid detection of false concave points. Given the sequence of extracted contour points $C=\left\{c_{1}, c_{2}, \ldots\right\}$, the dominant points are determined by co-linear suppression. To be specific, every contour point $c_{i}$ is examined for co-linearity, while it is compared to the previous and the next successive contour points. The point $c_{i}$ is considered as a dominant point if it is not located on the line connecting $c_{i-1}=\left(x_{i-1}, y_{i-1}\right)$ and $c_{i+1}=\left(x_{i+1}, y_{i+1}\right)$ and the distance $d_{i}$ from $c_{i}$ to the line connecting $c_{i-1}$ to $c_{i+1}$ is larger than a pre-set threshold $d_{i}>d_{t h}$.

Here, the $d_{t h}$ value was selected automatically using the method of Prasad et al. [15]. In this method, the threshold value $d_{t h}$ is selected automatically based on the angular distance between the slope of the actual line and the digitized line. After polygonal approximation and dominant point detection, the dominant point $c_{d, i} \in C_{d o m}$ is considered to be a concave point if [26]

$$
C_{c o n}=\left\{c_{d, i} \in C_{d o m}: \overrightarrow{c_{d, i-1} c_{d, i}} \times \overrightarrow{c_{d, i} c_{d, i+1}}>0\right\}
$$

\subsection{Segment Grouping}

Due to overlaps between objects and irregularities of the object shapes, a single object may produce multiple contour segments. Segment grouping is needed to merge all of the contour segments belonging to the same object. Let $S=\left\{S_{1}, S_{2}, \ldots, S_{N}\right\}$ be an ordered set of $N$ contour segments in a connected component of an image. The aim is to group the contour segments into $M$ subsets such that the contour segments that belong to individual objects are grouped together, and so that $M \leq N$.

Let $\omega_{i}$ be the group membership indicator giving the group index to which each contour segment $S_{i}$ belongs to. Denote $\Omega$ as the ordered set of all membership indicators: $\left\{\omega_{1}, \omega_{2}, \ldots, \omega_{N}\right\}$. The grouping criterion is given by a scalar function $J(\cdot)$ of $\Omega$ which maps a possible grouping of the given contour segments onto the set of real numbers $\mathbb{R} . J$ is the cost of grouping that ideally measures how the grouping $\Omega$ resembles the true contour segments of the objects. The grouping problem for the given set of $S$ is to 
find the optimal membership set $\Omega^{*}$ such that the grouping criterion (cost of grouping) is minimized as

$$
\Omega^{*}=\underset{\Omega}{\operatorname{argmin}} J(\Omega ; S) .
$$

The grouping task is formulated as a combinatorial optimization problem which is solved using the branch and bound (BB) algorithm [24]. The BB algorithm can be applied with any grouping criterion $J$. However, the selection of the grouping criterion has a significant effect on the overall performance. The proposed grouping criterion is a hybrid cost function consisting of two parts [24]: 1) the generic part ( $\left.J_{\text {concavity }}\right)$ that encapsulates the general convexity properties of the objects and 2) the specific part that encapsulates the properties of objects that are exclusive to a certain application, e.g., symmetry $\left(J_{\text {symmetry }}\right)$, and ellipticity $\left(J_{\text {ellipticity }}\right)$ as follows:

$$
J=\underbrace{J_{\text {concavity }}}_{\text {Generic }} \underbrace{+\beta J_{\text {ellipticity }}+\gamma J_{\text {symmetry }}}_{\text {Specific }}
$$

where $\beta, \gamma$ are the weights for each term respectively.

The generic part encourages the convexity assumption of the objects to penalize the grouping of the contour segments belonging to different objects. This is achieved by incorporating a quantitative concavity measure. Given two contour segments $s_{i}$ and $s_{j}$, the generic part of the cost function is defined as

$$
\mathrm{J}_{\text {concavity }}=\left(\frac{A_{s_{i} \cup s_{j}}-A_{\mathrm{ch}, s_{i} \cup s_{j}}}{A_{S_{i} \cup S_{j}}}\right)
$$

where $A_{s_{i} \cup s_{j}}$ is the area of a region bounded by $s_{i}, s_{j}$, and $A_{\mathrm{ch}, s_{i} \cup s_{j}}$ is the upper bound on the area of any convex hull with contour points $s_{i}$ and $s_{j}$.

The specific part is adapted to consider the application criteria and certain object properties. Considering the object under examination, several functions can be utilized. The ellipticity term measures the discrepancy between the fitted ellipse and the contour segments [26]. Given the contour segment $S_{i}$ consisting of $n$ points, $s_{i}=$ $\left\{\left(x_{k}, y_{k}\right)\right\}_{k=1}^{n}$, and the corresponding fitted ellipse points, $s_{f, i}=\left\{\left(x_{f, k}, y_{f, k}\right)\right\}_{k=1}^{n}$, the ellipticity term is defined as follows:

$$
\mathrm{J}_{\text {ellipticity }}=\frac{1}{n} \sum_{k=1}^{n} \sqrt{\left(x_{k}-x_{f, k}\right)^{2}+\left(y_{k}-y_{f, k}\right)^{2}} .
$$

The symmetry term penalizes the resulting objects that are non-symmetric. Let $o_{i}$ and $o_{j}$ be the centers of symmetry of the contour segments $s_{i}$ and $s_{j}$ obtained by aggregating the normal vector of the contour segments. The procedure applied is similar to the fast radial symmetry transform [8], but the gradient vectors are replaced by the normal vectors of the contour segments. This transform is referred to as the normal symmetry transform (NST).

In NST, every contour segment point gives a vote for the plausible radial symmetry at some specific distance from that point. Given the distance value $n$ of the predefined 
range $\left[R_{\min } R_{\max }\right]$, for every contour segment point $(x, y)$, NST determines the negatively affected pixels $P_{-e}$ by

$$
P_{-v e}(x, y)=(x, y)-\operatorname{round}\left(\frac{\boldsymbol{n}(x, y)}{\|\boldsymbol{n}(x, y)\|} n\right),
$$

and increments the corresponding point in the orientation projection image $\boldsymbol{O}_{n}$ by 1 . The symmetry contribution $\boldsymbol{S}_{n}$ for the radius $n \in\left[R_{\min }, R_{\max }\right]$ is formulated as

$$
\boldsymbol{S}_{n}(x, y)=\left(\frac{\left|\tilde{\boldsymbol{O}}_{n}(x, y)\right|}{k_{n}}\right)
$$

where $k_{n}$ is the scaling factor that normalizes $\boldsymbol{O}_{n}$ across different radii. $\tilde{\boldsymbol{O}}_{n}$ is defined as

$$
\tilde{\boldsymbol{O}}_{n}(x, y)= \begin{cases}\boldsymbol{O}_{n}(x, y), & \text { if } \boldsymbol{O}_{n}(x, y)<k_{r} \\ k_{n}, & \text { otherwise }\end{cases}
$$

The full NST transform $S$, by which the interest symmetric regions are defined, is given by the average of the symmetry contributions over all the radii $n \in\left[R_{\min }, R_{\max }\right]$ considered as

$$
\boldsymbol{S}=\frac{1}{|N|} \sum_{n \in\left[R_{\min }, R_{\max }\right]} \boldsymbol{S}_{n}
$$

The centers of symmetry $o_{i}$ and $o_{j}$ of the contour segments are estimated as the average locations of the detected symmetric regions in $S$. The symmetry term $J_{\text {symmetry }}$ is defined as the Euclidean distance between $o_{i}(x, y)$ and $o_{j}(x, y)$ as

$$
\mathrm{J}_{\text {symmetry }}=\left|o_{i}-o_{j}\right|
$$

This distance is normalized to $[0,1]$ by diving it by the maximum diameter of the object.

\subsection{Contour Estimation}

Once the contour evidences have been obtained, contour estimation is carried out to infer the missing parts of the overlapping NPs. A widely known approach is Least Square Ellipse Fitting (LSEF) [3]. The purpose of LSEF is to compute the ellipse parameters by minimizing the sum of squared algebraic distances from the known contour evidences points to the ellipse.

\section{Experiments}

\subsection{Data}

The experiments were carried out using a dataset from an interesting real-world application [6]. The dataset consists of BF TEM images of nanocatalyst NPs, present in cathode of a proton exchange fuel cell captured in a JEOL $2010 \mathrm{~F}$ operated $200 \mathrm{kV}$. This 
dataset contains 150 images, each of $1024 \times 1024$ pixels, containing a total of 2700 NPs. It should be noted that, in the original publication [6], the total number of NPs was reported as 2000. This is due to the fact that the paper considered only non-overlapping NPs.

As the quantity and diversity of the training samples are important factors in attempting to achieve high accuracy and only a small amount annotated images of NPs are available, we employ data augmentation to create a larger training dataset for image binarization. The augmented data were generated using affine transformations (rotation, shifts, and shearing). An expert annotated 20 binary masks consists of around 700 particles from the dataset. For training purposes in the U-Net framework around 1000 images were generated by data augmentation. The rest of Images in the dataset, 130, containing 2000 NPs was used as a final test set for binarization. The training images were zero-centered and normalized to unit variance. The network was trained for 20 epochs using the Adam optimization algorithm with a fixed initial rate of $1 \mathrm{e}-4$ and a mini-batch size of 8 patches in Keras. To asses the performance of U-Net, the training was performed using 10-Fold Cross Validation and the best performing model was recorded on each epoch. The model achieved the average accuracy of $98 \% \pm 0.001$, precision of $97 \% \pm 0.002$ and recall of $97 \% \pm 0.003$.

\subsection{Performance Measure}

To evaluate and to compare performances of the methods, the four different metrics were used. The detection performance was measured using the following three metrics: the true positive rate (TPR), the positive predictive value (PPV), and the accuracy (ACC), defined as follows:

$$
\begin{array}{r}
T P R=\frac{T P}{T P+F N}, \\
P P V=\frac{T P}{T P+F P}, \\
A C C=\frac{T P}{T P+F P+F N},
\end{array}
$$

where true positive (TP) is the number of correctly detected NPs, false positive (FP) is the number of incorrect segmentation results, and false negative $(\mathrm{FN})$ is the number of missed NPs.

To compute the number of correct or incorrect detection results, the Jaccard similarity coefficient (JSC) [19] was used. JSC was chosen since it interprets the similarity by the ratio of overlap between the ground truth NP and the segmented NP. JSC considers the amount of the common area between two NPs. Since we are often interested in area measurements this is an appropriate metric. Given the binary feature model of the segmented object $I_{p}$ and the ground truth particle $I_{g}$, JSC is computed as

$$
J S C=\frac{\left|I_{p} \cap I_{g}\right|}{\left|I_{p} \cup I_{g}\right|} .
$$

The threshold value for the ratio of overlap (JSC threshold) was set to 0.6. In this way, if the area of overlap between segmented object $I_{p}$ and the ground truth particle $I_{g}$ 
was more than $60 \%$ then the segmented object considered as TP. The average JSC (AJSC) and DSC (ADSC) value were used as another measure of the segmentation and binarization performance.

\subsection{Parameter Selection}

The proposed method requires the following major parameters:

Weighting parameters $\beta, \gamma$ define to what extent the ellipticity and symmetry features must be weighted in the computation of the grouping cost $J$. A lower value emphasizes non-ellipticity and non-symmetry features where higher values ensure that objects have perfect elliptical symmetry shapes. This parameter is useful when the objects are not perfectly elliptical or symmetry. In this work, NPs have perfect elliptical symmetry shapes; thus the symmetry and ellipticity terms were weighted equally to 0.5 .

Radial range $\left[R_{\min }, R_{\max }\right]$ determines the range of radii at which the NST transform $S$ is computed. The radial range should be defined as such that it covers the variety of all objects sizes available in the image. Considering the smallest and largest object axes, the radial ranges was set $R_{\min }=18$ and $R_{\max }=22$ for our NPs dataset.

\subsection{Results}

Binarization The U-Net binarization methods were compared to six competing methods: hybridized variance (HV) [6], Otsu [13] Kittler [7], Niblack [12], Sauvola [18], and Bresen [1]. The results of the image binarization methods applied to the Inorganic fuel cell datasets is presented in Table 1. From the result it can be seen that the proposed U-Net achieves the highest ADSC and AJSC scores compare to the competing methods. Fig. 2 shows an example of image binarization methods applied to a BF TEM image containing NPs. As it can be seen in Fig. 2, the U-Net performs better as compared to the competing methods. This shows the superiority of U-Net which can handle various type of image degradation such as noise, non uniform background and uneven-illumination.

Table 1: Comparison of performances of binarization methods on the fuel cell NPs dataset. The best method based on each criterion is shown bolded.

U-Net [17] HV [6] Otsu [13] Kittler [7] Niblack [12]Sauvola [18] Bresen [1]

\begin{tabular}{llllllll}
\hline AJSC & $\mathbf{9 3}$ & 88 & 62 & 67 & 28 & 66 & 26 \\
ADSC & $\mathbf{9 6}$ & 93 & 72 & 78 & 42 & 78 & 41 \\
\hline
\end{tabular}

Segmentation The proposed NPs segmentation methods were compared to five existing methods for overlapping NPs segmentation: hybridized variance (HV) [6], seed point-based contour evidence extraction (SCC) [20], concave point-based contour evidence extraction (CC) [22], nanoparticle segmentation (NPA) [14], and concave points extraction and contour segmentation (CECS) [26]. These methods were chosen since they have been previously successfully applied to the segmentation of NPs images. It 


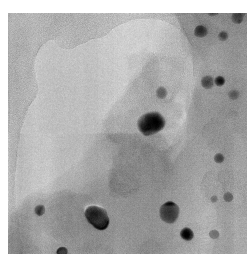

(a)

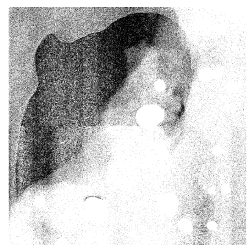

(e)

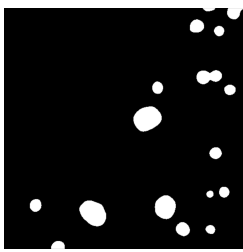

(b)

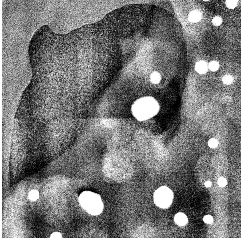

(f)

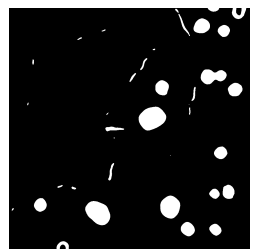

(c)

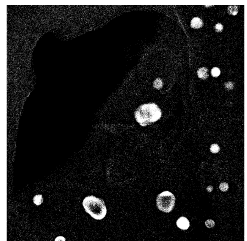

(g)

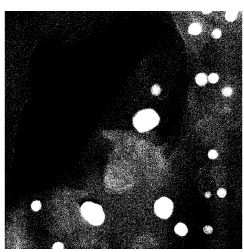

(d)

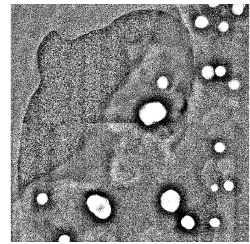

(h)

Fig. 2: An example of image binarization methods: (a) Original image; (b) U-Net [17]; (c) HV [6]; (d) Otsu [13]; (e) Kittler [7] (f) Niblack [12]; (g) Sauvola [18]; (h) Bernsen [1].

should be noted that, due to the fact that the generic image binarization was not considered in [20|22|14|26], the proposed U-Net binarization method was applied also for SCC,CC,NPA and CECS methods.

Example results of these segmentation methods applied to an image of the fuel cell NPs dataset are shown in Fig. 3 As it can be seen, HV, CC, and NPA suffered from under-segmentation, while SCC and CECS experienced over-segmentation. The CC and CECS methods failed to achieve acceptable results on NPs whose shapes deviated substantially from an ellipse. The NPA and SCC methods failed to work when the sizes of the NPs varied widely. Our proposed method achieved satisfactory segmentation results and outperformed the other methods in this regard. Table 2 presents the corresponding performance statistics of the competing segmentation methods when applied to the Inorganic fuel cell NPs dataset. The segmentation results show that the proposed method outperforms the other methods, achieving higher scores of TPR, PPV, and ACC. Fig. 4 4 represents the effect of the Jaccard similarity threshold on the TPR, PPV, and ACC scores of the proposed segmentation and competing methods. The performance of all segmentation methods degraded when the JSC threshold was increased, while our proposed segmentation method was better than the other methods, achieving higher JSC threshold values.

\section{Conclusions}

This paper presents a novel method for automated segmentation of NPs in BF TEM images. The proposed method consists of the following three main steps: the image binarization to divide the image into foreground (nanoparticles) and background, the contour evidence extraction to detect the visible part of each nanoparticle, and the contour 
Table 2: Comparison of performances of segmentation methods on the fuel cell NPs dataset. The best method based on each criterion is shown bolded.

\begin{tabular}{lcccc}
\hline Methods & $\begin{array}{c}\text { TPR } \\
{[\%]}\end{array}$ & $\begin{array}{c}\text { PPV } \\
{[\%]}\end{array}$ & ACC & AJSC \\
& {$[\%]$} \\
\hline Proposed & $\mathbf{9 0}$ & $\mathbf{9 2}$ & $\mathbf{8 5}$ & $\mathbf{8 7}$ \\
HV [6] & 89 & 91 & 83 & 85 \\
CC [22] & 81 & 87 & 74 & 82 \\
SCC [20] & 83 & 87 & 74 & 86 \\
NPA [14] & 80 & 87 & 73 & 87 \\
CECS [26] & 88 & 73 & 68 & 86 \\
\hline
\end{tabular}

estimation to estimate the nanoparticle contours. The binarization is performed using the well known U-Net deep encoder-decoder framework. The contour evidence extraction is performed by detecting concave points and finding contour segment groupings using the branch and bound algorithm. Finally, the contour estimation is performed using ellipse fitting. The experiments showed that the proposed method reduces the false negatives and false positives relative to all of the compared methods, while increasing the number of individual NPs segmented from regions of overlap. The proposed method achieves high detection and segmentation accuracies, and outperforms five competing methods on the real dataset of NPs images.

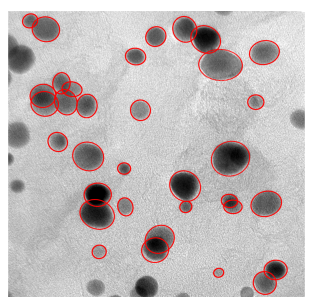

(a)

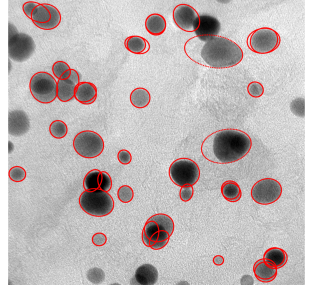

(d)

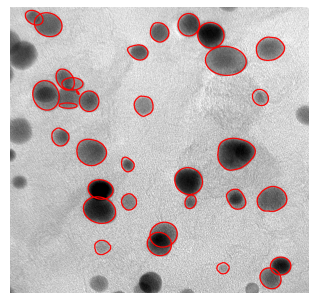

(b)

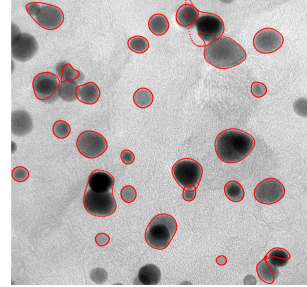

(e)

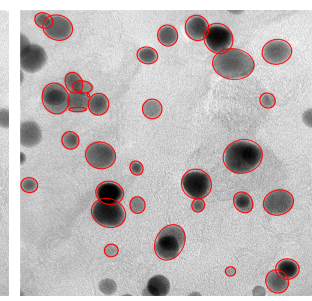

(c)

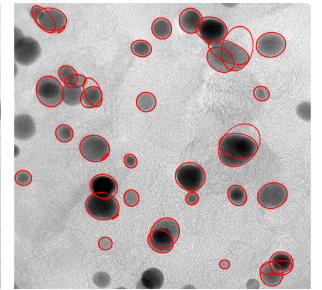

(f)

Fig. 3: Example results of the segmentation methods applied to an image of fuel cell NPs: (a) Proposed; (b) HV; (c) CC; (d) SCC; (e) NPA; (f) CECS. 


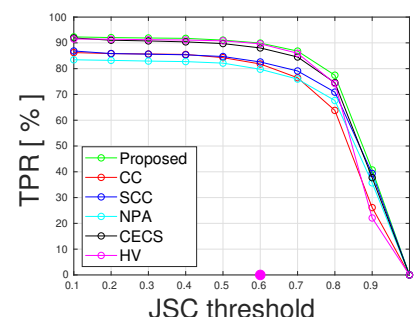

JSC threshold

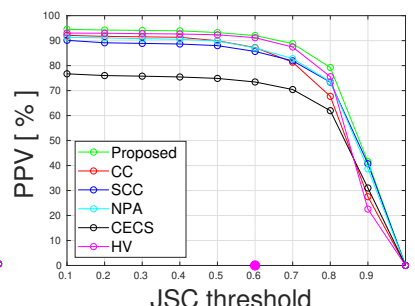

JSC threshold

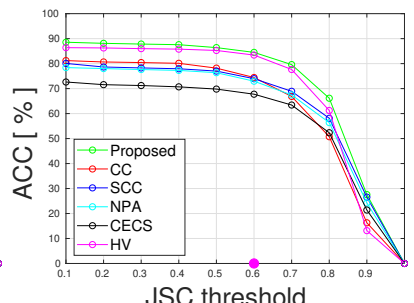

JSC threshold

Fig. 4: Effect of different JSC threshold values on segmentation performance on the fuel cell NPs dataset: (a) TPR; (b) PPV; (c) ACC.

\section{Acknowledgments}

The research was carried out in the Computational Photonics Imaging (COMPHI) and the Automatic segmentation of overlapping objects for cell image analysis (CellVision) projects. The authors would like to thank Academy of Finland for funding the CelIVision project (Decision No. 313598). The authors would like to acknowledge Prof. Heikki Haario and Dr. Jouni Sampo from the LUT University and Dr. Daniel Groom from University of Texas for their collaboration. Moreover, the authors would like to thank support of the fuel cell technologies office of the US Department of Energy (DoE), office of energy efficiency and renewable energy. Dr. Nancy Garland was the DoE technology development manager for this work. The work was subcontracted by Argonne National Laboratory, a DoE, office of science laboratory operated under contact no. DE-AC02-06CH1135 by U.Chicago, Argonne, LLC, DE-AC02-07CH11358.

\section{References}

1. Bernsen, J.: Dynamic thresholding of grey-level images. In: Proceedings of the Eighth International Conference on Pattern Recognition (ICPR). pp. 1251-1255 (2009)

2. Fisker, R., Carstensen, J., Hansen, M., Bødker, F., Mørup, S.: Estimation of nanoparticle size distributions by image analysis. Journal of Nanoparticle Research 2(3), 267-277 (Sep 2000)

3. Fitzgibbon, A., Pilu, M., Fisher, R.B.: Direct least square fitting of ellipses. IEEE Transactions on Pattern Analysis and Machine Intelligence 21(5), 476-480 (1999)

4. Gilbert, J.A., Kariuki, N.N., Wang, X., Kropf, A.J., Yu, K., Groom, D.J., Ferreira, P.J., Morgan, D., Myers, D.J.: Pt catalyst degradation in aqueous and fuel cell environments studied via in-operando anomalous small-angle x-ray scattering. Electrochimica Acta 173, 223 - 234 (2015)

5. Gontard, L.C., Ozkaya, D., Dunin-Borkowski, R.E.: A simple algorithm for measuring particle size distributions on an uneven background from tem images. Ultramicroscopy 111(2), $101-106(2011)$

6. Groom, D., Yu, K., Rasouli, S., Polarinakis, J., Bovik, A., Ferreira, P.: Automatic segmentation of inorganic nanoparticles in bf tem micrographs. Ultramicroscopy 194, 25 - 34 (2018) 
7. Kittler, J., Illingworth, J.: Minimum error thresholding. Pattern Recognition 19(1), 41-47 (1986)

8. Loy, G., Zelinsky, A.: Fast radial symmetry for detecting points of interest. IEEE Transactions on Pattern Analysis and Machine Intelligence 25(8), 959-973 (2003)

9. Melander, M., Latsa, V., Laasonen, K.: Co dissociation on iron nanoparticles: Size and geometry effects. The Journal of Chemical Physics 139(16), 164320 (2013)

10. Miller, J., Kropf, A., Zha, Y., Regalbuto, J., Delannoy, L., Louis, C., Bus, E., van Bokhoven, J.: The effect of gold particle size on auau bond length and reactivity toward oxygen in supported catalysts. Journal of Catalysis 240(2), 222-234 (2006)

11. Murray, C.B., Kagan, C.R., Bawendi, M.G.: Synthesis and characterization of monodisperse nanocrystals and close-packed nanocrystal assemblies. Annual Review of Materials Science 30(1), 545-610 (2000)

12. Niblack, W.: An Introduction to Digital Image Processing. Strandberg Publishing Company, Birkeroed, Denmark, Denmark (1985)

13. Otsu, N.: A threshold selection method from gray-level histograms. Automatica 11(285296), 23-27 (1975)

14. Park, C., Huang, J.Z., Ji, J.X., Ding, Y.: Segmentation, inference and classification of partially overlapping nanoparticles. IEEE Transactions on Pattern Analysis and Machine Intelligence 35(3), 669-681 (2013)

15. Prasad, D.K., Leung, M.K.: Polygonal representation of digital curves. INTECH Open Access Publisher (2012)

16. Pyrz, W.D., Buttrey, D.J.: Particle size determination using tem: a discussion of image acquisition and analysis for the novice microscopist. Langmuir 24(20), 11350-11360 (2008)

17. Ronneberger, Olafand Fischer, P., Brox, T.: U-net: Convolutional networks for biomedical image segmentation. In: Medical Image Computing and Computer-Assisted Intervention (2015)

18. Sauvola, J., Pietikäinen, M.: Adaptive document image binarization. Pattern Recognition 33(2), 225-236 (2000)

19. Taha, A.A., Hanbury, A.: Metrics for evaluating $3 \mathrm{~d}$ medical image segmentation: analysis, selection, and tool. In: BMC Medical Imaging (2015)

20. Zafari, S., Eerola, T., Sampo, J., Kälviäinen, H., Haario, H.: Segmentation of overlapping elliptical objects in silhouette images. IEEE Transactions on Image Processing 24(12), 59425952 (Dec 2015)

21. Zafari, S.: Segmentation of Partially Overlapping Convex Objects in Silhouette Images. Ph.D. thesis, Lappeenranta University of Technology (2018)

22. Zafari, S., Eerola, T., Sampo, J., Kälviäinen, H., Haario, H.: Segmentation of partially overlapping nanoparticles using concave points. In: Advances in Visual Computing (2015)

23. Zafari, S., Eerola, T., Sampo, J., Kälviäinen, H., Haario, H.: Comparison of concave point detection methods for overlapping convex objects segmentation. In: Scandinavian Conference on Image Analysis. pp. 245-256 (2017)

24. Zafari, S., Eerola, T., Sampo, J., Kälviäinen, H., Haario, H.: Segmentation of partially overlapping convex objects using branch and bound algorithm. In: ACCV 2016 International Workshops. pp. 76-90 (2017)

25. Zafari, S., Murashkina, M., Eerola, T., Sampo, J., Kälviäinen, H., Haario, H.: Resolving overlapping convex objects in silhouette images by concavity analysis and gaussian process. arXiv preprint arXiv:1906.01049 (2019)

26. Zhang, W.H., Jiang, X., Liu, Y.M.: A method for recognizing overlapping elliptical bubbles in bubble image. Pattern Recognition Letters 33(12), 1543-1548 (2012)

27. Zhang, W., Li, R., Deng, H., Wang, L., Lin, W., Ji, S., Shen, D.: Deep convolutional neural networks for multi-modality isointense infant brain image segmentation. NeuroImage 108, $214-224(2015)$ 